\title{
Value distribution of difference polynomials
}

\author{
By Ilpo LAINE*) and Chung-Chun YANG**) \\ (Communicated by Shigefumi MorI, M.J.A., Oct. 12, 2007)
}

\begin{abstract}
We continue to studying value distribution of difference polynomials of meromorphic functions. In particular, we show that extending classical theorems of TumuraClunie type to difference polynomials needs additional assumptions.
\end{abstract}

Key words: Nevanlinna theory; difference polynomial; Clunie lemma; Tumura-Clunie theorem.

1. Introduction. The classical Clunie lemma [3] may be formulated as follows, see [5; Theorem 3.9]:

Lemma A. Let $f$ be a transcendental meromorphic solution of

$$
f^{n} P(z, f)=Q(z, f)
$$

where $P(z, f), Q(z, f)$ are differential polynomials in $f$ and its derivatives with small meromorphic coefficients $a_{\lambda}, \lambda \in I$, in the sense of $m\left(r, a_{\lambda}\right)=$ $S(r, f)$ for all $\lambda \in I$. If the total degree of $Q(z, f)$ as a polynomial in $f$ and its derivatives is $\leq n$, then

$$
m(r, f)=S(r, f) .
$$

The Clunie lemma has been a powerful tool in the field of complex differential equations and related fields. In particular, the lemma has been used to studying value distribution of certain differential polynomials. We first recall two versions of what are presently known as TumuraClunie type theorems, see [5; Theorem 3.9] and [7; Corollary on p. 115]:

Theorem B. Suppose that $f$ is meromorphic and non-constant in the complex plane, that

$$
g(z):=f(z)^{n}+P_{n-1}(z, f)
$$

where $P_{n-1}(z, f)$ is a differential polynomial of total degree at most $n-1$ in $f$ and its derivatives with small coefficients $a(z)$ in the sense of $T(r, a)=$ $S(r, f)$, and that

$$
N(r, f)+N\left(r, \frac{1}{g}\right)=S(r, f)
$$

2000 Mathematics Subject Classification. Primary 30D35 $39 \mathrm{~A} 05$.

*) Department of Mathematics, University of Joensuu, FI80101 Joensuu, Finland.

**) Department of Mathematics, Hong Kong University of Science and Technology, Hong Kong, China.
Then

$$
g(z)=(f(z)+\alpha(z))^{n},
$$

where $T(r, \alpha)=S(r, f)$.

Theorem C. Let $f$ be a meromorphic function, and suppose that

$$
\Psi(z):=a_{n} f(z)^{n}+\cdots+a_{0}(z)
$$

has small meromorphic coefficients $a_{j}(z), a_{n} \neq 0$, in the sense of $T\left(r, a_{j}\right)=S(r, f)$. Moreover, assume that

$$
\bar{N}\left(r, \frac{1}{\Psi}\right)+\bar{N}(r, f)=S(r, f)
$$

Then

$$
\Psi=a_{n}\left(f+\frac{a_{n-1}}{n a_{n}}\right)^{n}
$$

For our reasoning below, we need the following modification of Theorem C.

Theorem D. Let $f$ be a meromorphic function of finite order $\rho$, and suppose that

$$
\Psi(z):=a_{n} f(z)^{n}+\cdots+a_{0}(z)
$$

has small meromorphic coefficients $a_{j}(z), a_{n} \neq 0$ in the sense of $T\left(r, a_{j}\right)=O\left(r^{\rho-1+\varepsilon}\right)+S(r, f)$. Moreover, assume that

$$
\bar{N}\left(r, \frac{1}{\Psi}\right)+\bar{N}(r, f)=O\left(r^{\rho-1+\varepsilon}\right)+S(r, f) .
$$

Then

$$
\Psi=a_{n}\left(f+\frac{a_{n-1}}{n a_{n}}\right)^{n} .
$$

Proof. The proof is, with apparent modifications, the same as the proof of Theorem C in [7].

Recently, Halburd-Korhonen [4] and Chiang- 
Feng [1] investigated the value distribution theory of difference expressions. A key result, see [1; Corollary 2.6], of these studies to be applied below reads as follows:

Lemma E. Given two distinct complex constants $\eta_{1}, \eta_{2}$, let $f$ be a meromorphic function of finite order $\rho$. Then, for each $\varepsilon>0$, we have

$$
m\left(r, \frac{f\left(z+\eta_{1}\right)}{f\left(z+\eta_{2}\right)}\right)=O\left(r^{\rho-1+\varepsilon}\right) .
$$

As for difference counterparts of the Clunie lemma, see [4; Corollary 3.3]. We recall the following more general version, see [6; Theorem 2.3], corresponding to [8; Theorem 1]:

Theorem F. Let $f$ be a transcendental meromorphic solution of finite order $\rho$ of a difference equation of the form

$$
U(z, f) P(z, f)=Q(z, f) .
$$

where $U(z, f), P(z, f), Q(z, f)$ are difference polynomials such that the total degree deg $U(z, f)=n$ in $f(z)$ and its shifts $f\left(z+c_{1}\right), \ldots, f\left(z+c_{k}\right)$, and deg $Q(z, f) \leq n$. Moreover, assume that all coefficients $b_{\lambda}$ in (2) are small in the sense that $T\left(r, b_{\lambda}\right)=S(r, f)$ and that $U(z, f)$ contains exactly one term of maximal total degree in $f(z)$ and its shifts. Then, for each $\varepsilon>0$,

$$
m(r, P(z, f))=O\left(r^{\rho-1+\varepsilon}\right)+S(r, f),
$$

possibly outside of an exceptional set of finite logarithmic measure.

A natural question is now whether some kind of difference counterpart of Tumura-Clunie type theorems can be proved. However, Theorem 1 below seems to show that such a direct counterpart cannot be found. To formulate our result, take a difference polynomial

$$
G(z)=\sum_{\lambda \in J} b_{\lambda}(z) \prod_{j=1}^{\tau_{\lambda}} f\left(z+\delta_{\lambda, j}\right)^{\mu_{\lambda, j}},
$$

where

$$
\max _{\lambda \in J} \sum_{j=1}^{\tau_{\lambda}} \mu_{\lambda, j}=n
$$

and at least one of the shift arguments $\delta_{\lambda, j}$ is nonzero. Moreover, we assume that the coefficients in (4) are small in the sense of $T\left(r, b_{\lambda}\right)=O\left(r^{\rho-1+\varepsilon}\right)+$ $S(r, f)$.

Theorem 1. If $f$ is a meromorphic function of finite order $\rho$ such that

$$
N(r, 1 / f)+N(r, f)=O\left(r^{\rho-1+\varepsilon}\right)+S(r, f),
$$

the difference polynomial (4) in $f(z)$ and its shifts, of maximal total degree $n$, must have sufficiently many zeros to satisfy

$$
N(r, 1 / G) \neq O\left(r^{\rho-1+\varepsilon}\right)+S(r, f) .
$$

Remark. Observe that whenever two of the counting functions $N(r, 1 / f), N(r, f), N(r, 1 / G)$ are of growth $O\left(r^{\rho-1+\varepsilon}\right)+S(r, f)$ at most, then the third one is $\neq O\left(r^{\rho-1+\varepsilon}\right)+S(r, f)$. This immediately follows from the proof of Theorem 1 below.

Concerning value distribution of difference products, we prove

Theorem 2. Let $f(z)$ be a transcendental entire function of finite order, and $c$ be a non-zero complex constant. Then for $n \geq 2, f(z)^{n} f(z+c)$ assumes every non-zero value $a \in \mathbf{C}$ infinitely often.

2. Proof of Theorem 1. Contrary to the assertion, we assume that

$$
N(r, 1 / G)=O\left(r^{\rho-1+\varepsilon}\right)+S(r, f) .
$$

To prove Theorem 1, we propose to follow the idea in the proof of [6; Theorem 2.3]. Therefore, we transform (4) into a polynomial expression of $f(z)$ by writing $(f(z+\delta) / f(z)) f(z)$ in place of the shifts $f(z+\delta)$ of $f(z)$. The expression (4) then takes the form

$$
G(z)=\sum_{j=0}^{n} \widetilde{b}_{j}(z) f(z)^{j},
$$

where each of the coefficients $\widetilde{b}_{j}(z), j=1, \ldots, n$ is the sum of finitely many terms of type

$$
b_{\lambda}(z) \prod_{j=1}^{\tau_{\lambda}}\left(f\left(z+\delta_{\lambda, j}\right) / f(z)\right)^{\mu_{\lambda, j}} .
$$

By Lemma E and our assumption concerning the coefficients $b_{\lambda}$, we immediately see that

$$
m\left(r, \widetilde{b}_{j}\right)=O\left(r^{\rho-1+\varepsilon}\right)+S(r, f)
$$

for $j=1, \ldots, n$. Moreover, by the [1; Theorem 2.2],

$$
N\left(r, \widetilde{b}_{j}\right)=O\left(r^{\rho-1+\varepsilon}\right)+S(r, f)
$$

and so

$$
T\left(r, \widetilde{b}_{j}\right)=O\left(r^{\rho-1+\varepsilon}\right)+S(r, f)
$$

for $j=0, \ldots, n$. By Theorem $\mathrm{D}$, we may write

$$
G(z)=\widetilde{b}_{n}(f(z)+\alpha(z))^{n},
$$

where $T(r, \alpha)=O\left(r^{\rho-1+\varepsilon}\right)+S(r, f)$. Therefore, by 
assumption, we have

$$
N\left(r, \frac{1}{f+\alpha}\right)=O\left(r^{\rho-1+\varepsilon}\right)+S(r, f) .
$$

An application of the second main theorem for small target functions implies $T(r, f)=O\left(r^{\rho-1+\varepsilon}\right)+$ $S(r, f)$, a contradiction.

Remark. The restriction in Theorem 1 to the finite order case seems to be essential. In fact, it is easy to find entire functions of infinite order having no zeros such that a differential polynomial of type (4) in $f$ and its shifts has no zeros. As an example, take $f(z)=\exp \left(e^{z}\right)$ and consider $G(z):=f(z)^{2}+f(z+c)$, where $c$ satisfies $e^{c}=2$. Then we have $G(z)=2 f(z)^{2}=2 \exp \left(2 e^{z}\right)$. Moreover, this example shows that a difference counterpart to Theorem B cannot hold, at least not in the case of infinite order. Indeed, we have $N(r, f)+$ $N(r, 1 / G)=O\left(r^{\rho-1+\varepsilon}\right)+S(r, f)$. If $G(z)=2 f(z)^{2}=$ $(f(z)+\alpha(z))^{2}$ for a meromorphic function $\alpha(z)$ small with respect to $f(z)$, then $f(z)^{2}=$ $2 \alpha(z) f(z)+\alpha(z)^{2}$, which implies that $T(r, \alpha)=$ $T(r, f)+O(1)$, a contradiction. Another similar example is given by $f(z)=\exp \left(\frac{1}{4} \sin ^{2} z\right)$ that satisfies

$$
G(z):=\exp \left(\sin ^{2} z\right)=f^{4}(z)+f(z) f\left(z+\frac{\pi}{2}\right)-e^{1 / 4} .
$$

We now have

$$
N(r, f)+N(r, 1 / f)+N(r, 1 / G)=S(r, f) .
$$

If $G(z)=(f(z)+\alpha(z))^{4}$ for a small function $\alpha(z)$, then

$$
4 f^{3}+6 \alpha f^{2}+4 \alpha^{2} f+\alpha^{3}=0,
$$

and so $T(r, f)=O(T(r, \alpha))=S(r, f)$, a contradiction.

3. Proof of Theorem 2. Suppose, contrary to the assertion, that

$$
f(z)^{n} f(z+c)-a=p(z) e^{q(z)}
$$

where $p(z), q(z)$ are polynomials, not vanishing identically. Differentiating (5) and eliminating $e^{q(z)}$, we obtain

$$
f(z)^{n-1} P(z, f(z))=-a p^{*}(z),
$$

where

$$
\begin{gathered}
P(z, f(z))=n p(z) f^{\prime}(z) f(z+c) \\
+p(z) f(z) f^{\prime}(z+c)-p^{*}(z) f(z) f(z+c),
\end{gathered}
$$

and

$$
p^{*}(z)=p^{\prime}(z)+p(z) q^{\prime}(z) .
$$

First observe that $P(z, f(z))$ cannot vanish identically. Indeed, if $P(z, f(z)) \equiv 0$, then $p^{*}(z) \equiv 0$. Since $p(z), q(z)$ are polynomials, we immediately conclude that they are constants. Therefore, $f(z)^{n} f(z+c)$ is a constant as well, and so

$(n+1) T(r, f)=(n+1) m(r, f)=$
$(n+1) m\left(r, \frac{f(z)}{f(z+c)}\right)+O(1)=O\left(r^{\rho-1+\varepsilon}\right)+S(r, f)$

by Lemma E, a contradiction. To apply now Lemma A, we rewrite $P(z, f(z))$ as

$$
\begin{gathered}
P(z, f(z))=n p(z) \frac{f(z+c)}{f(z)} f(z) f^{\prime}(z) \\
+p(z) \frac{f^{\prime}(z+c)}{f(z+c)} f(z) f(z+c)-p^{*}(z) f(z) f(z+c) .
\end{gathered}
$$

Then a simple modification of Lemma A, together with [4; Corollary 3.3], implies that

$$
T(r, P(z, f))=O\left(r^{\rho-1+\varepsilon}\right)+S(r, f)
$$

and

$$
T(r, f P(z, f))=O\left(r^{\rho-1+\varepsilon}\right)+S(r, f),
$$

hence $T(r, f)=O\left(r^{\rho-1+\varepsilon}\right)+S(r, f)$, a contradiction.

Remark. Theorem 2 does not remain valid, if $n=1$. Indeed, take $f(z)=1+e^{z}$. Then

$$
f(z) f(z+\pi i)-1=\left(1+e^{z}\right)\left(1-e^{z}\right)-1=-e^{2 z} .
$$

Moreover, the assertion of Theorem 2 may fail, if $f(z)$ is of infinite order. This can be seen by taking $f(z)=\exp \left(e^{z}\right)$. Then $f(z) f(z+\pi i)=1$ and so

$$
f(z)^{2} f(z+\pi i)=f(z)
$$

has no zeros.

4. Discussion. It remains open whether a difference counterpart to Theorem B holds for meromorphic functions of finite order. A possible strategy to obtain a restricted variant of TumuraClunie might be to assume that $f$ is of order $\rho<1$, and to apply the idea in [2; Theorem 4.1], to transform the difference polynomial (4) into a differential polynomial. To this end, denote

$$
\beta_{c}(z):=\frac{f(z+c)}{f(z)}-1-c \frac{f^{\prime}(z)}{f(z)},
$$

for all shifts of $f(z)$ to be applied. The expression (4) then takes the form 
(6) $\quad G=\sum_{\lambda \in J} b_{\lambda} \prod_{j=1}^{\tau_{\lambda}}\left(1+\delta_{\lambda, j} \frac{f^{\prime}}{f}+\beta_{\lambda, j}\right)^{\mu_{\lambda, j}} f^{\mu_{\lambda, j}}$.

By [2; Theorem 4.1], we know that each of the proximity functions $m\left(r, \beta_{\lambda, j}\right)$ in (6) satisfies $m\left(r, \beta_{\lambda, j}\right)=(\rho-1+\varepsilon) \log r$ for all radii $r$ outside of an exceptional set of finite logarithmic measure. However, there seems to be no way to show that the counting function for the coefficients in (6) would be small (in the Nevanlinna theory sense), due to the zeros of $f$. Therefore, this strategy does not seem to be very promising.

Acknowledgements. The authors were supported by the RGC Grant of Hong Kong 605205. The first author has been also supported by the Academy of Finland grant 210245.

\section{References}

[ 1 ] Y.-M. Chiang and S.-J. Feng, On the Nevanlinna characteristic of $f(z+\eta)$ and difference equa- tions in the complex plane, Ramanujan J. (to appear).

[ 2 ] Y.-M. Chiang and S.-J. Feng, On the growth of logarithmic differences, difference quotients and logarithmic derivatives of meromorphic functions, arXiv:math.CV/0610480. (Preprint).

[ 3 ] J. Clunie, On integral and meromorphic functions, J. London Math. Soc. 37 (1962), 17-27.

[ 4 ] R. Halburd and R. Korhonen, Difference analogue of the lemma on the logarithmic derivative with applications to difference equations, J. Math. Anal. Appl. 314 (2006), 477-487.

[ 5 ] W. Hayman, Meromorphic Functions, Clarendon Press, Oxford, 1964.

[ 6 ] I. Laine and C.-C. Yang, Clunie theorems for difference and q-difference polynomials, J. London Math. Soc. (to appear).

[ 7 ] E. Mues and N. Steinmetz, The theorem of Tumura-Clunie for meromorphic functions, J. London Math. Soc. (2) 23 (1981), 113-122.

[ 8 ] C.-C. Yang and Z. Ye, Estimates of the proximate function of differential polynomials, Proc. Japan Acad. Ser. A Math. Sci. 83 (2007), 5055 . 